Bundesgesundheitsbl - Gesundheitsforsch Gesundheitsschutz 2004 - 47:36-40 DOI 10.1007/s00103-003-0761-8
In der Diskussion

J. Bertram · M. Mielke · M. Beekes · K. Lemmer · M. Baier · G. Pauli Robert Koch-Institut, Berlin

\section{Inaktivierung und Entfernung von Prionen bei der Aufbereitung von Medizinprodukten}

\author{
Ein Beitrag zur Prüfung und Deklaration \\ geeigneter Verfahren
}

Zu den Aufgaben des Robert Koch-Institutes (RKI) gehört es, gesundheitliche Risiken zu identifizieren, zu analysieren und Maßnahmen bzw. Empfehlungen zu ihrer Minimierung zu erarbeiten und zu kommunizieren. Dementsprechend wurde in Reaktion auf die BSE-Problematik ${ }^{1}$ in einer umfangreichen Arbeitsgruppe beim RKI der Bericht der "Task Force vCJK" zur Minimierung des Risikos einer iatrogenen Übertragung von Prionen durch chirurgische Instrumente erarbeitet und im April 2002 auf der Basis der damaligen Kenntnis veröffentlicht [1]. Danach stellt eine geeignete alkalische Reinigung mit anschließender Dampfsterilisation bei $134^{\circ} \mathrm{C}$ ein routinefähiges Verfahren zur Minimierung des Übertragungsrisikos von Prionen durch chirurgische Instrumente dar. Vorliegende Ergebnisse zur inaktivierenden Wirkung anderer Verfahren wurden bisher mit unterschiedlichen Prüfmethoden gewonnen und nach individuellen Kriterien bewertet. Wir teilen deshalb die Meinung von Experten, dass eine Vereinheitlichung der Prüfmethoden erforderlich ist [2], nicht zuletzt um die Entwicklung weiterer für die Routine und spezielle Anforde-

\footnotetext{
${ }^{1}$ BSE ist die bovine, spongiforme Enzephalopathie, deren Erreger, auf den Menschen übertragen, die Variante der CreutzfeldtJakob-Krankheit (vCJK) auslösen können. Als Verursacher von transmissiblen spongiformen Enzephalopathien (TSE) gelten Prionen (proteinaceous infectious particles).
}

rungen geeigneter Verfahren zur Dekontamination von Prionen zu fördern. Wir möchten hier Anforderungen an die Prüfung und Deklaration entsprechender Verfahren zur Diskussion stellen. Ausdrücklich wird darauf hingewiesen, dass in allen Fällen eines erkennbaren Risikos für das Vorliegen einer transmissiblen spongiformen Enzephalopathie (TSE) den bestehenden Empfehlungen des RKI [1,3] zu folgen ist.

ie sichere Aufbereitung von Medizinprodukten stellt einen besonders sensiblen Bereich der Infektionsprävention im Rahmen der Krankenhaushygiene dar. Anforderungen an die Hygiene bei der Aufbereitung von Medizinprodukten sind in der gleich lautenden gemeinsamen Empfehlung von der Kommission für Krankenhaushygiene und Infektionsprävention beim RKI und dem BfArM vom 20. August 2001 formuliert [4].

Ein bisher nicht zufrieden stellend gelöstes Problem stellen potenziell oder unerkannt mit Prionen kontaminierte Medizinprodukte dar: Prionen sind aufgrund ihrer physikalisch-chemischen Eigenschaften schwer von Oberflächen abzulösen und zu inaktivieren. Werden chirurgische Instrumente oder andere Medizinprodukte an Patienten mit erkennbarem Risiko für CJK, vCJK oder sonstige humane TSE angewandt, gelten deshalb besondere Empfehlungen. Im
April 2002 erschien in Reaktion auf die BSE-Problematik die Mitteilung des RKI über die Variante der Creutzfeldt-JakobKrankheit (vCJK) unter besonderer Berücksichtigung der Prävention einer iatrogenen Übertragung durch chirurgische Instrumente $[1,5,6,7]$.

In dem Task-Force-Bericht [1] wird das Vorgehen bei erkennbarem Risiko (mögliche oder klinisch wahrscheinliche vCJK) sowie bei nicht erkennbarem Risiko beschrieben. Für den Fall eines erkennbaren Risikos wird auf die RKIEmpfehlungen von 1998 zum Vorgehen bei CJK-Verdacht [3] verwiesen mit der Ergänzung einer ggf. erforderlichen sicheren Asservierung der Instrumente bis zur Klärung der Diagnose im Verdachtsfall. In allen anderen Fällen ohne erkennbares Risiko soll zur Aufbereitung von Medizinprodukten eine Kombination von wenigstens 2 auch für die Entfernung/Inaktivierung von Prionen zumindest partiell geeigneten Verfahren eingesetzt werden.

Nicht zuletzt aufgrund von Erkenntnissen, die die Auslösung von im Phänotyp klassischen CJK-Formen durch BSE möglich erscheinen lassen [8, 9], und dem in der Schweiz beobachte-

(c) Springer-Verlag 2004

Dr.J.Bertram

Robert Koch-Institut,

Nordufer 20,13353 Berlin

E-Mail:bertramj@rki.de 
Tabelle 1

Übersicht über Prüfphasen und daraus ableitbare Deklarationen

\begin{tabular}{|c|c|c|}
\hline Phase 1a & Phase 1b & Phase 2 \\
\hline $\begin{array}{l}\text { Vorprüfung von Verfahren in vitro } \\
\text { im qualitativen Suspensionstest; } \\
\text { Auswertung z. B. mittels Westernblot }\end{array}$ & $\begin{array}{l}\text { Quantitativer Suspensionstest } \\
\text { Auswertung im Tierversuch }\end{array}$ & $\begin{array}{l}\text { Quantitativer Carrier-Test } \\
\text { Auswertung im Tierversuch }\end{array}$ \\
\hline Basistest, keine Deklaration & $\begin{array}{l}\text { Deklaration als } \\
\text { Prion-inaktivierend }\end{array}$ & $\begin{array}{l}\text { In Verbindung mit bestan- } \\
\text { dener Phase } 1 \mathrm{~b} \text { Deklaration } \\
\text { als Prion-dekontaminierend }\end{array}$ \\
\hline
\end{tabular}

Ggf. kann bereits in Phase 1a ein In-vitro-Test an Carriern ausgeführt werden. Eine solche Prüfvariante liegt neueren Arbeiten zu diesem Thema zugrunde [35]. Das Verfahren beruht darauf, dass mit PrPSc haltigem Hirnhomogenat beschichtete Stahlstifte der Testlösung unter definierten Bedingungen ausgesetzt werden. Nach der Einwirkungszeit wird sowohl der auf dem Stahlstift verbliebene als auch der abgelöste Anteil des Hirnhomogenates auf mit Proteinase Knicht abbaubares PrPSc im Westernblot geprüft. Aufgrund des Prüfdesigns lassen sich aus den Ergebnissen Aussagen zur Prionen abbauenden oder destabilisierenden sowie zur Prionen ablösenden Wirkung treffen ten Anstieg von CJK-Fällen hat die dortige Regierung eine „Verordnung über die Prävention der Creutzfeldt-JakobKrankheit bei chirurgischen und medizinischen Eingriffen" [10] erlassen. ${ }^{2}$

Bisher bekannt zuverlässige Verfahren zur Inaktivierung von Prionen, z. B. die Behandlung in 2,5\% Natriumhypochlorit- oder in $1 \mathrm{M}$ Natriumhydroxidlösung mit anschließender oder kombinierter Dampfsterilisation $[1,11,12]$, sind in der Routine nicht oder nur eingeschränkt anwendbar [2].

Zur Temperaturempfindlichkeit von Prionen ist anzumerken, dass bei Dampfsterilisation selbst unter Anwendung von 18 Minuten Sterilisierzeit bei $134-137^{\circ} \mathrm{C}$ ein gewisser Anteil der Prionen infektiös bleiben kann $[1,13]$ und trockene Hitze sogar bei extremen Temperaturen eine Restinfektiosität nicht beseitigt hat [14]. Für verbesserte Reinigungs- und Inaktivierungsverfahren besteht somit ein dringender Bedarf.

\footnotetext{
2 Explizit wird in amtlichen Erläuterungen zu dieser Verordnung angeführt:,,Vor diesem Hintergrund (schwer einschätzbares iatrogenes Infektionsrisiko) und in Anbetracht der langen Inkubationszeit von erworbenen menschlichen Prion-Erkrankungen müssen gewisse Präventionsmaßnahmen im Sinne des Vorsorgeprinzips sofort ergriffen werden, ohne im Detail abschätzen zu können, ob das Ausmaß ihres Einsatzes gerechtfertigt ist. Im Interesse ihrer Optimierung müssen sie jedoch auf der Basis von entsprechenden Forschungsarbeiten kontinuierlich neu evaluiert und in angemessener Weise angepasst werden."
}

\section{Allgemeine Anforderungen an geeignete Aufbereitungs- verfahren}

Gemäß den „Anforderungen an die Hygiene bei der Aufbereitung von Medizinprodukten“ [4] erfolgt die Aufbereitung von Medizinprodukten in der Regel in Stufen,

Deginnend mit einer sachgerechten Vorbereitung,

Defolgt von einer gründlichen Reinigung und Spülung,

D vor abschließender Desinfektion, Spülung und Trocknung bzw. Sterilisation.

In Anbetracht der besonderen physikalisch-chemischen Eigenschaften von Prionen ist auf eine geeignete Vorbereitung des Reinigungsgutes mit dem Ziel, die Fixierung von infektiösem Material zu vermeiden, besonders zu achten $[15,16$, 17, 18]. Desinfektionsmittel, die z. B. aus Gründen des Personenschutzes in dieser Phase der Aufbereitung verwendet werden, sollten mit Blick auf das nachfolgende Reinigungsverfahren so ausgewählt werden, dass eine Fixierung von Proteinrückständen auf oder an den Medizinprodukten vermieden wird $[4,19]$.

Aus praktischen Gründen ist ein einfaches, wenn möglich maschinelles Reinigungsverfahren anzustreben, an das im Einzelnen folgende Anforderungen zu stellen sind:

Döglichst universelle Einsetzbarkeit, D keine wesentliche Änderung des gewohnten Ablaufes der Aufbereitung,
D Vermeidung von Kreuzkontaminationen,

D Dekontamination, bevor Verschmutzungen wie Proteine antrocknen/fixiert werden können,

D Materialverträglichkeit, z. B. gegenüber Stahl, Aluminium und Kunststoffpolymeren,

D geringe Schaumbildung bei maschineller Reinigung oder Ultraschallanwendung.

Reinigungsverfahren, bei denen die eingesetzten Reinigungsmittel gleichzeitig mit der Entfernung der Erreger zumindest teilweise deren Inaktivierung bewirken, ist insbesondere wegen der Kreuzkontaminationsproblematik der Vorzug zu geben. Für sehr empfindliche Instrumente/Medizinprodukte (mit Optiken, Klebungen etc.) können produktspezifische mehrstufige Verfahren mit jeweils partiell wirksamen Einzelschritten nötig sein.

Die eingesetzten Verfahren sollen ökologisch und toxikologisch unbedenklich sein. Die Wirksamkeit entsprechender Verfahren zur Inaktivierung und Entfernung von Prionen muss durch geeignete Prüfungen belegt sein [1]. Diesbezüglich wird der folgende Vorschlag zu Wirksamkeitsnachweis und Deklaration unterbreitet.

\section{Wirksamkeitsnachweis und Deklaration}

Die Prüfung und Deklaration der Wirksamkeit eines Verfahrens zur Dekontamination ${ }^{3}$ von Prionen sollte in Anlehnung an die Wirksamkeitsprüfung bakterizider und viruzider Agenzien in Phasen erfolgen. Allerdings gestaltet sich der Nachweis der Inaktivierung des infektiösen Agens bei Prionen schwieriger, da bisher kein einfaches biologisches Testverfahren zur Verfügung steht. Bereits veröffentlichte Verfahren zur Inaktivierung von Prionen $[20,21,22,23,24,25,26$, 27,28 ] sind bisher nicht nach einheitli-

\footnotetext{
3 In der Richtlinie zur Krankenhaushygiene (Urban Fischer Verlag, München/Jena) ist der Begriff Dekontamination definiert als , $_{\text {ein }}$ Verfahren zur Abreicherung/Reduktion von organischen Verunreinigungen und gleichzeitiger oder aufeinander folgender Inaktivierung von Infektionserregern im Rahmen einer standardisierten Reinigung/ Desinfektion".
} 
chen Kriterien bewertet worden. Gegenwärtig kann die Inaktivierung von Prionen abschließend nur durch Tierversuche belegt werden $[1,20]$. Die Untersuchungen sollten so angelegt sein, dass quantitative Aussagen über die Abreicherung/Titer-Reduktion möglich sind.

\section{Prüfanschmutzungen}

Als infektiöses Ausgangsmaterial sollten Materialien verwendet werden, die in einem tierexperimentellen System hinsichtlich beobachtbarer Effekte gut etabliert sind und eine den humanmedizinisch relevanten Prionen ähnliche oder höhere Resistenz haben. Bisher wird häufig der Scrapie-Stamm $263 \mathrm{~K} \mathrm{im}$ Hamstermodell verwendet $[20,21]$. Um Matrixeinflüsse [13] auf die Resistenz zu berücksichtigen, wird für die Prüfungen in der Regel Hirnhomogenat, z. B. 10\% Hirnhomogenat mit einem Titer von $\geq 10^{7} \mathrm{LD}_{50} / \mathrm{ml}$ verwendet.

\section{Prüfkörper}

Als Prüfkörper für Carrier-Teste kommen bevorzugt solche in Frage, die nach Kontamination mit geeignetem Material (s. Kapitel „Prüfanschmutzungen") sowohl für In-vitro- als auch Invivo-Untersuchungen geeignet sind $[29,30]$.

\section{Nachweis der Inaktivierung und Entfernung von Prionen}

International standardisierte Methoden liegen für die Prüfung der Prion-dekontaminierenden Wirksamkeit bisher nicht vor. Nach gegenwärtigem Wissensstand schlagen wir deshalb ein mehrstufiges Vorgehen vor:

D in Phase 1 einen qualitativen (Phase $1 \mathrm{a}$ ) und quantitativen (Phase $1 \mathrm{~b}$ ) Suspensionstest und

D in Phase 2 einen für den Tierversuch geeigneten Carrier-Test.

Im Einzelnen (s. Tabelle 1) bedeutet dies

D in Phase 1a die Vorprüfung geeigneter Substanzen bzw. Verfahren durch den In-vitro-Nachweis, dass durch diese Substanzen oder Verfahren $\operatorname{PrP} P^{S c} 4$ abgebaut oder zumindest dessen Struktur destabilisiert wird (Basistest),
D in Phase $1 \mathrm{~b}$ den Nachweis und die Quantifizierung der inaktivierenden Wirkung ausgewählter Substanzen bzw. Verfahren im Tierversuch durch intrazerebrale Verimpfung [31,32, 33] behandelten, infektiösen Materials (s. Abschnitt Prüfanschmutzungen) (Suspensionstest),

D in Phase 2 den Nachweis der dekontaminierenden Wirkung ausgewählter Substanzen bzw. Verfahren, geprüft auf relevanten Oberflächen von Prüfkörpern, die für die intrazerebrale Applikation im Tierversuch geeignet sind (wie z. B. Stahlstifte $[29,30]$ ).

\section{Bewertung der Ergebnisse und Deklaration}

Die Phase 1a ist bestanden, wenn nach Einwirkung des zu prüfenden Mittels im Suspensionstest durch geeignete Analyseverfahren, z. B. im Westernblot, kein $\mathrm{PrPSc}^{\mathrm{Sc}}$ mehr nachweisbar ist [34].

Die Phase $1 b$ ist bestanden, wenn im Tierversuch nach intrazerebraler Verimpfung behandelten infektiösen Materials eine statistisch gesicherte Abreicherung um mindestens 4 log-Stufen nachweisbar ist, die Tiere der Testgruppe nach Ablauf der doppelten Inkubationszeit einer gleichzeitig mitgeführten Kontrollgruppe (in der unbehandeltes infektiöses Material verimpft wurde) keine Anzeichen einer Erkrankung durch Prionen erkennen lassen und zu diesem Zeitpunkt kein $\operatorname{PrP}^{\mathrm{Sc}}$ im Gehirn dieser Tiere nachweisbar ist (z. B. histochemisch oder im Westernblot). Verfahren bzw. Mittel, welche die Prüfungen in Phase $1 \mathrm{~b}$ bestanden haben, können als Prion-inaktivierend bezeichnet werden.

Die Phase 2 ist bestanden, wenn nach intrazerebraler Implantation von kontaminierten und behandelten Prüfkörpern die Tiere der Testgruppe nach

\footnotetext{
${ }^{4}$ Zur Erläuterung häufig verwendeter $\mathrm{Ab}$ kürzungen: PrP: Prion-Protein; PrPC:zelluläres PrP, nicht mit Infektiosität assoziiert und sensitiv gegenüber Proteinase $\mathrm{K}$; $\mathrm{PrPSc}$ : pathologische Isoform des PrP, mit Infektiosiät assoziiert und partiell resistent gegen Proteinase K; PrP 27-30: mit Infektiosität assoziierter Proteinase-K-resistenter Kern des PrPSc; PrPRES: fehlgefaltete Form des PrionProteins, biochemisch partiell resistent gegen Proteinase $K$, aber nicht notwendigerweise mit Infektiosität assoziiert. Weitere Begriffe und Abkürzungen zur Prionen- bzw. PrP-Nomenklatur s. Literatur [28], S. 526 f.
}

Ablauf der doppelten Inkubationszeit einer gleichzeitig mitgeführten Kontrollgruppe (in der unbehandelte kontaminierte Prüfkörper implantiert wurden) keine Anzeichen einer Erkrankung durch Prionen erkennen lassen und zu diesem Zeitpunkt kein $\mathrm{PrP}^{\mathrm{Sc}}$ im Gehirn dieser Tiere nachweisbar ist (z. B. histochemisch oder im Westernblot). Verfahren, die zusätzlich zur Phaseıb auch Phase 2 bestanden haben, können als Priondekontaminierend bezeichnet werden (s. Tabelle 1).

\section{Aspekte zur Verfahrensentwicklung}

Anhaltspunkte für die Entwicklung geeigneter Verfahren können neben speziell mit der Prion-Inaktivierung befassten Veröffentlichungen z. B. Arbeiten zu folgenden Fragestellungen liefern:

D Proteinadsorption bzw. -desorption an verschiedenen Oberflächen [36, $37,38]$,

D Löslichkeit von Proteinen [39, 40, 41, $42,43,44,45,46,47,48,49,50$ ],

D Struktur und physikalisch-(bio-)chemische Eigenschaften von Prionprotein $[51,52,53,54,55,56,57]$,

Derteilungsverhalten von $\operatorname{PrP}^{\mathrm{Sc}}[58]$.

In Ergänzung zur Behandlung mit feuchter Hitze (Dampfsterilisation) sind folgende Ansätze zur Inaktivierung von Prionen denkbar:

Dasische Agenzien wie $\mathrm{NaOH}$ und $\mathrm{KOH}[11,12,34,35]$,

D stark oxidierende Mittel,

D oberflächenaktive Substanzen (Tenside), zwitterionische Amphotenside oder andere waschaktive Substanzen wie Phosphate bzw. Phosphonate,

D chaotrope Substanzen wie Guanidiniumthiocyanat (GdnSCN),

D Disulfid spaltende Reduktionsmittel wie Thiole (DTT),

D hitzebeständige, proteolytisch oder anderweitig inaktivierende Enzyme $[59,60]$,

D mit $\mathrm{PrP}^{\mathrm{Sc}}$ unter Einschluss oder Anlagerung reagierende Substanzen, wie z. B. tricyclische Verbindungen oder Farbstoffe [61, 62, 63],

D " $\beta$-sheet-breaker" [64].

Ein Teil dieser Stoffe oder Wirkprinzipien ist bereits als unwirksam oder nur 
partiell wirksam beurteilt worden, allerdings auf der Basis von Ergebnissen, die unter unterschiedlichen experimentellen Bedingungen gewonnen wurden. Einige dieser Stoffe könnten jedoch in Kombination mit wirksamen Stoffen additiv nützlich sein. Generell wäre es für Reiniger aus mehreren Bestandteilen wichtig, in Phase 1a eine sorgfältige Kompatibilitätsprüfung der Einzelkomponenten vorzunehmen, da deren Wirkung sich aufheben, aber auch ergänzen oder verstärken kann.

\section{Literatur}

1. Abschlussbericht der Task Force VCJK (2002) Die Variante der Creutzfeldt-Jakob-Krankheit (vCJK). Bundesgesundheitsbl Gesundheitsforsch Gesundheitsschutz 45:376-394

2. McDonnell G, Burke $P$ (2003) The challenge of prion decontamination. CID 36:1152-1154

3. RKI-Mitteilung (1998) Krankenversorgung und Instrumentensterilisation bei CJK-Patienten und CJK-Verdachtsfällen. Bundesgesundheitsbl Gesundheitsforsch Gesundheitsschutz 41:279-285

4. Empfehlungen der Kommission für Krankenhaushygiene und Infektionsprävention (2001) Anforderungen an die Hygiene bei der Aufbereitung von Medizinprodukten. Bundesgesundheitsbl Gesundheitsforsch Gesundheitsschutz 44:1115-1126

5. Informationen zu CJK/vCJK, http://www.rki.de, dort Link zu Gesundheit und Krankheiten, weiterer Link zur Krankenhaushygiene

6. Beekes M, Mielke M, Kurth R (2002) Aspekte zur Risikoabschätzung und Prävention nosokomialer Übertragungen der klassischen und varianten Creutzfeldt-Jakob-Krankheit. Internist 43:738,741-748

7. Beekes $M$, Mielke M, Pauli $G$ et al. (in press) Aspects of risk assessment and risk management of nosocomial transmission of classical and variant Creutzfeldt-Jakob disease with special attention to German regulations. In: Rabenau HF, Cinatl J, Doerr HW (eds) Prions. A Challenge for science, medicine and public health system. 2nd, revised edition. Contrib Microb, vol 11. Basel, Karger, pp 117-135

8. Asante EA et al. (2002) BSE prions propagate as either variant CJD-like or sporadic CJD-like prion strains in transgenic mice expressing human prion protein. EMBO J 21:6358-6366

9. Butler $D$ (2002) Prion data suggest BSE link to sporadic CJD. Nature 420:450

10. http://www.bag.admin.ch/prionen/d/ verordn.d.pdf

11. Taguchi F et al. (1991) Proposal for a procedure for complete inactivation of the CreutzfeldJakob disease agent. Arch Virol 119:297-301

12. Ernst DR, Race RE (1993) Comparative analysis of scrapie agent inactivation methods. J Virol Methods 41:193-202
13. Appel TR et al. (2001) Heat stability of prion rods and recombinant prion protein in water, lipid and lipid-water mixtures.J Gen Virol 82:465-473

14. Taylor DM, McConnell I, Fernie K (1996) The effect of dry heat on the ME7 strain of mouse-passaged scrapie agent.J Gen Virol 77:3161-3164

15. Taylor DM (1999) Inactivation of prions by physical and chemical means.J Hosp Infect 43 [Suppl]:S69-S76

16. WHO (1999) Infection control guidelines for transmissible spongiform encephalopathies. Report of a WHO Consultation, Geneva, Switzerland, 23-26 March 1999 (WHO/CDS/CSR/APH/2000.3); http://www. who.int/emc-documents/tse/whocdscsraph2003c.html, link PDF/english

17. Iffenecker $A$, Ruef C (2002) Übertragungsrisiko von Prionen:Stellungnahme zur Aufbereitung thermostabiler chirurgischer Instrumente vor der Sterilisation. Swiss NOSO 9:25-28

18. Rutala WA, Weber DJ (2001) Creutzfeldt-Jakob disease: recommendations for disinfection and sterilisation. Clin Infect Dis 32:1348-1356

19. Bloß R (2002) Einfluss der Desinfektionswirkstoffe auf die Instrumentenreinigung. http://www.bode-chemie, Link zu Service/ Publikationen/Instrumentenaufbereitung

20. Taylor DM (2000) Inactivation of transmissible degenerative encephalopathy agents: a review. Vet J 159:10-17

21. Taylor DM et al. (1994) Decontamination studies with the agents of bovine spongiform encephalopathy and scrapie. Arch Virol 139:313-326

22. Shenoy VN, Revak TT (1998) Prion inactivation in connective tissue materials. Off Gaz US Patent Trademark off pat 1210:3648

23. Reichl H (1997) Methods of inactivating prions (slow viruses) conventional viruses and other infections agents in biological material. Off Gaz US Patent Trademark off pat 1198:2591

24. Prusiner SB et al. (1993) Attempts to restore scrapie prion infectivity after exposure to protein denaturants. Proc Nat Acad Sci 90:2793-2797

25. Chartier V et al.(2001) Experimental results of detergent and pre-disinfection treatments before sterilisation of medical devices. Zentralsterilisation 9:100-107

26. Antloga $\mathrm{K}$ et al. (2000) Prion disease and medical devices. ASAI0 46:69-72

27. Yamamoto $\mathrm{M}$ et al. (2001) Glycidol degrades scrapie mouse prion protein. J Vet Med Sci 63:983-990

28. Hörnlimann B, Leutwiler A, Oberthür RC, Widmer HR (2001) Die chemische Desinfektion und Inaktivierung von Prionen. In: Hörnlimann B, Riesner D, Kretzschmar H (Hrsg) Prionen und Prionkrankheiten. de Gruyter, Berlin-New York, S 381-388, 389

29. Zobeley E et al. (1999) Infectivity of scrapie prions bound to a stainless steel surface. Mol Med 5:240-243

30. Flechsig E et al. (2001) Transmission of scrapie by steel-surface-bound prions. Mol Med 7:679-684
31. Kimberlin RH, Walker CA (1986) Pathogenesis of scrapie (strain $263 \mathrm{~K}$ ) in hamsters infected intracerebrally, intraperitoneally or intraocularly. J Gen Virol 67:255-263

32. Kimberlin RH, Walker CA (1977) Characteristics of a short incubation model of scrapie in the golden hamster.J Gen Virol 34:295-304

33. Prusiner SB et al. (1982) Measurement of the scrapie agent using an incubation time interval assay. Ann Neurol 11:353-358

34. Käsermann F, Kempf C (2003) Sodium hydroxide renders the prion protein $\mathrm{PrPSc}^{\mathrm{Sc}}$ sensitive to proteinase K.J Gen Virol 84:3173-3176

35. Lemmer K, Mielke M, Pauli G, Beekes M (2003) Prion decontamination of surgical instruments: in vitro studies on the destabilization, degradation and removal of surface-bound PrPSC. In: International Prion Conference „Prion Diseases: from basic research to intervention concepts"; München, 8.10.-10.10.2003: Abstract IV-02

36. Sharma CP, Chandy T (1987) Influence of L-ascorbic acid, blood cells and components on protein adsorption/desorption on polycarbonate. Haemostasis 17:70-78

37. Williams DF, Askill IN, Smith R (1985) Protein absorption and desorption phenomena on clean metal surfaces.J Biomed Mat Res 19:313-320

38. Biswas SC, Chattoraj DK (2002) Kinetics of desorption of preadsorbed proteins from the surface of silica and charcoal by various desorbing reagents. http://preprint. chemweb.com/chemeng/0206003

39. Narhi LO et al. (1997) Fractionating and characterization of polyclonal antibodies using three progressively more chaotropic solvents. Anal Biochem 253:246-252

40. Washabaugh MW, Collins KD (1986) The systematic characterization by aqueous column chromatography of solutes which affect protein stability.J Biol Chem 261:12477-12485

41. Stöckel J, Hartl FU (2001) Chaperonin-mediated de novo generation of prion protein aggregates.J Mol Biol 313:861-872

42. DebBurman SK, Lindquist S (2000) Chaperonepromoted solubilization of protease-resistant prion protein. FASEB J 14:425

43. Hjelmeland LM (1990) Solubilization of native membrane proteins. Meth Enzym 182:253-264

44. Xiong LW et al. (2001) Conformational change, aggregation and fibril formation induced by detergent treatments of cellular prion protein. J Neurochem 79:669-679

45. Herbert B (1999) Advances in protein solubilisation for two-dimensional electrophoresis. Electrophoresis 20:660-663

46. Fountoulakis M, Takacs B (2001) Effect of strong detergents and chaotropes on the detection of proteins in two-dimensional gels. Elektrophoresis 22:1593-1602

47. König S, Schmidt O, Rose K et al. (2003) Sodium dodecyl sulfate versus acid-labile surfactant gel electrophoresis: comparative proteomic studies on rat retina and mouse brain. Electrophoresis 24:751-756

48. Ross ARS et al. (2002) Identification of proteins from two-dimensional polyacrylamide gels using a novel acid-labile surfactant. Proteomics 2:928-936 
49. Chevallet M et al. (1998) New zwitterionic detergents improve the analysis of membrane proteins by two-dimensional electrophoresis. Electrophoresis 19:1901-1909

50. Arbab ME, Tinay AH (1997) Effect of cooking and treatment with sodium bisulphite or ascorbic acid on the in vitro protein digestibilty of two sorghum cultivars. Food Chem 59:339-343

51. Madec JY et al. (1997) Biochemical properties of protease resistant prion protein $\mathrm{PrPSc}$ in natural sheep scrapie. Arch Virol 142:1603-1612

52. Warwicker J, Cole C (2001) The structural and spectroscopic basis for modelling prion protein interactions. Spectroscopy 15:151-159

53. Herrmann LM, Caughey B (1998) The importance of the disulfide bond in prion protein conversion. Neuroreport 9:2457-2461

54. Brown DR, Clive C, Haswell SJ (2001) Antioxidant activity related to copper binding of native prion protein. J Neurochem 76:69

55. Feughelman M, Willis B (2002) Potential involvement of copper and thiol-disulphide interchange in prion proteins' conformational conversion. Medical Hypotheses 59:321

56. Martins V, Brentani R (2002) The biology of the cellular prion protein. Neurochem Int 41:353

57. Callahan MA, Xiong L, Caughey B (2001) Reversability of scrapie-associated prion protein aggregation.J Biol Chem 276:28022-28028

58. Lee DC et al. (2001) A direct relationship between the partitioning of the pathogenic prion protein and transmissible spongiform encephalopathy infectivity during the purification of plasma proteins. Transfusion 41:449-455

59. PL976065: Inactivation of the causative agents of transmissible spongiform encephalopathies by thermophilic and hyperthermophilic proteases. http://tse-ip.org/eu-projects/ pl976065-eu-tse-project.shtml

60. http://www.newscientist.com/news/ news.jsp?id=ns99993999 29 July 03

61. Prusiner BS, Safar JG (2001) Removal of prions from blood, plasma and other liquids contacting with a solid substrate comprising a complexing agent such as sodium phosphotungstate to bind Prions in the sample, separating sample from the substrate. US Patent 6221614

62. Forloni $G$ et al. (2002) Tetracyclines affect prion infectivity. PNAS 99:10849

63. Demaimey R, Chesebro B, Caughey B (2000) Inhibition of formation of protease-resistant prion protein by Trypan Blue, Sirius Red and other Congo Red analogs. Arch Virol Suppl 16:277

64. Soto C et al. (2000) Reversion of prion protein conformational changes by synthetic betasheet breaker peptides. Lancet 355:192-197

\section{Neuer HTA-Bericht: Wie effektiv sind antivirale Therapien bei chronischer Hepatitis C?}

Die Deutsche Agentur für Health Technology Assessment des DIMDI, DAHTA@DIMDI, hat die medizinische und ökonomische Effektivität der Therapie von chronischer Hepatitis C mit Virostatika (Arzneimittel zur Therapie von Vireninfektionen) im Rahmen einer Modellierung bewertet. Das Ergebnis der Modellierung weist darauf hin, dass die Behandlung von chronischer Hepatitis $C$ mit Peginterferon und Ribavirin medizinisch und ökonomisch effektiver ist als die Standardtherapie mit Interferon und Ribavirin.

Durch Viren hervorgerufene Leberentzündungen gehören zu den weltweit häufigsten Infektionskrankheiten. Die Gesamtzahl der HCVInfizierten in Deutschland wird auf mindestens 400.000 geschätzt, von denen $80 \%$ eine chronische Hepatitis C entwickeln. Bei 20\% der chronisch Erkrankten entwickelt sich innerhalb von Jahrzehnten eine Leberzirrhose, verbunden mit einem erhöhten Risiko von Blutungen in der Speiseröhre, Ansammlung von Flüssigkeit in der Bauchhöhle, Verwirrtheit und Leberkrebs. Die Standardtherapie von chronischer Hepatitis C beruht auf einer Kombination von Interferon und Ribavirin. Derzeit wird jedoch auch eine Kombination aus Peginterferon und Ribavirin angewendet. Diese wird im Vergleich zur Standardtherapie effektiver eingeschätzt und vom Robert KochInstitut als optimal bezeichnet. Diese Behandlung ist jedoch kostenintensiver als der Standard.

Die Literaturrecherche offenbart einen Mangel an geeigneten Studien für eine Bewertung. Eine Modellierung auf Basis vorhandener Studien zeigt jedoch: Die Kombinationstherapie mit Peginterferon und Ribavirin führt zu einer um rund 4,5 Jahre höheren Lebenserwartung bei zuvor nicht behandelten Patienten mit erhöhten Transaminasewerten gegenüber einer Behandlung ohne Virostatika. Die Standardtherapie erhöht die Lebenserwartung um rund 2,5 Jahre und die Monotherapie mit Interferon noch um rund ein Jahr. Diese antiviralen Therapien verringern das Risiko, im Verlauf von 20 Jahren nach einer HCV-Infektion an einer Lebererkrankung zu sterben, um bis zu 46\%. Die PeginterferonRibavirin-Therapie verursacht gegenüber der Standardtherapie Mehrkosten, die aus volkswirtschaftlicher Sicht zweckmäßig scheinen, weil damit Kosten für Folgeerkrankungen vermieden werden können.
Nähere Informationen über Inhalt und Autoren des Berichts sind zu finden unter www.dimdi.de - Das DIMDI - Publikationen Schriftenreihen. Die Bücher aus der Schriftenreihe HTA sind im Buchhandel und über das DIMDI zum Preis von je 20,00 Euro (zzgl.Versandkosten) erhältlich. Bestellungen per E-Mail bei: huke@dimdi.de.

Ansprechpartner:

Dr. Hans-Peter Dauben,

Tel. (0221) 4724-311,

E-Mail:dauben@dimdi.de

Das Deutsche Institut für Medizinische Dokumentation und Information (DIMDI) in Köln stellt ein hochwertiges Informationsangebot für alle Bereiche des Gesundheitswesens zur Verfügung. Zu den Aufgaben des DIMDI gehören u.a. die Herausgabe deutscher Versionen von medizinischen Klassifikationen, der Aufbau von Informationssystemen für Arzneimittel, Medizinprodukte und Health Technology Assessment (HTA).

Buch zum Thema: Antivirale Therapie bei Patienten mit chronischer Hepatitis C in Deutschland. Medizinische und ökonomische Evaluation der initialen Kombinationstherapie mit Interferon/Peginterferon und Ribavirin. Siebert U, Sroczynski G unter Mitwirkung der German Hepatitis C Model (GEHMO) Group. HTA Schriftenreihe, Band 8, Hrsg.: Dauben, Rüther, Warda. Köln, 2003. ISBN 3-89906-726-6 